\title{
Juegos terribles: dificultad y nuevos excesos lúdicos
}

Diego Maté (1)

\begin{abstract}
Resumen: En los últimos años, en el videojuego se asentaron algunas recurrentes formas de exceso. Una modalidad ya común supone la exacerbación de la dimensión lúdica: juego tras juego, se proponen desafíos cada vez más difíciles de superar. La tensión entre esta nueva producción y la oferta mainstream sugiere la estabilización de operaciones relacionadas con el desborde. En otras esferas de la cultura, operaciones de ese tipo fueron analizadas a la luz de la categoría estética de lo sublime, sobre todo a partir del barroco en los lenguajes artísticos. Una zona de la producción independiente del videojuego está vehiculizando excesos lúdicos a través de diferentes estrategias textuales, como se vio con la expansión de los juegos estilo Souls, I Wanna be the Guy o los Kaizo Mario. Desde una perspectiva semiótica, la principal novedad de estos juegos tiene que ver con la regularización de una enunciación opaca que quiebra con las previsibilidades de los géneros y del diseño mainstream y vuelve ostensible los mecanismos discursivos. Esa opacidad se cifra sobre todo en la visibilización de un enunciador omnipresente, cuyo trabajo se revela constantemente en la sobreabundancia de desafíos, trampas y enigmas que los juegos despliegan. Este desplazamiento comunicativo supone una gran novedad en la oferta del medio, donde tradicionalmente parece haber dominado la transparencia enunciativa.
\end{abstract}

Palabras clave: enunciación - Dark Souls - exceso - sociosemiótica - performance - Kaizo Mario - abusive game design

[Resúmenes en inglés y portugués en las páginas 108-109]

(1) Licenciado en Crítica de Artes, maestrando en Crítica y Difusión de las Artes y doctorando en Artes, en los tres casos por la Universidad Nacional de las Artes (UNA).En su tesis doctoral investiga la producción del videojuego y sus cruces e intercambios con el campo de las artes desde la perspectiva de la sociosemiótica. Dirige el grupo de estudios de Juegos, Videojuegos y Game Studies (UNA). 


\section{Introducción}

En los últimos años, se dieron cambios discursivos que modificaron a gran escala el paisaje mediático del videojuego y que parecieran estar señalando la entrada a un nuevo momento histórico del dispositivo: aparición de una zona de producción independiente que toma distancia del mainstream y expande el repertorio expresivo del medio; surgimiento de los casual games, propuesta que reformula el contrato lúdico de los géneros tradicionales y que da lugar a un nuevo perfil de jugador; puesta en diálogo del videojuego con otras esferas de la cultura como la publicidad, el periodismo o la medicina, pero sobre todo con el arte, que reconfigura el lugar social del medio y sus modos de acceso. Esta efervescencia discursiva pareciera estar señalando la entrada a un nuevo período histórico. Los game studies no han dado casi periodizaciones, y las que están suscriben todas a un determinismo tecnológico que ordena la producción de cinco décadas en generaciones de consolas y dispositivos (Herman, 1993, Herz, 1997, Kent, 2001, Donovan, 2010). Propongo, en cambio, una periodización estilística que atienda a las transformaciones discursivas del videojuego: un momento inicial sin una norma que regule la producción textual y en la que el videojuego se referencia en otros medios y lenguajes (70); velozmente, los moldes de la previsibilidad social organizan la producción y aparecen los primeros géneros y estilos identificables (80); el desarrollo de los primeros motores gráficos en tres dimensiones posibilita una dominancia estilística: el realismo de espacios tridimensionales y texturas fotorrealistas tendrá de ahora en más el carácter de norma (90); finalmente, alrededor del nuevo milenio, la emergencia de nuevas soluciones formales (por ejemplo, la aparición de las estilísticas pixeladas) señala el fin de la dominancia del realismo y el paso a una etapa nueva en la que el videojuego abandona su posición privilegiada en el campo del entretenimiento y se integra a otros espacios sociales con sus prácticas (2000). Es en este cuarto y último momento donde se inscriben los cambios mencionados al inicio: cuando el videojuego entabla todo tipo de relaciones e intercambios con el campo de las artes. Una de esas relaciones consiste en la apropiación de operaciones originadas y difundidas en el campo de las artes (aunque no limitadas a él). La lista sería extensa, pero a modo de ejemplo, pueden mencionarse las estrategias tendientes a generar ambigüedad, ambivalencia e incertidumbre que actualizan la categoría de lo siniestro (Maté 2019b, 2019c). En el presente, muchos juegos despliegan formas del exceso que reenvían a la categoría de lo sublime.

\section{Acerca de lo sublime}

La noción de lo sublime se populariza con el libro del filósofo escocés Edmund Burke, XXX, en 1757. Allí, Burke ofrece una primera sistematización de lo sublime: se trata de un tipo de experiencia caracterizada por el terror, pero también de un conjunto de rasgos que la suscitan, por ejemplo, la oscuridad, la vastedad, el poder, el vacío o la soledad. Se plantea así una ambivalencia: si bien lo sublime es entendido como un tipo de experiencia, ya en Burke el concepto oscila entre la subjetividad y la descripción de aspectos materiales (Gras, 1998). A su vez, Burke piensa la categoría como formando sistema con lo bello. 
Para el autor, lo bello no corresponde a lo armónico y lo simétrico, sino a lo pequeño, lo liso y lo que posee "una variedad en la dirección de las partes" (Burke, 1998). Es decir, que ya en sus inicios, lo sublime funciona en tensión con otra cosa. No haré una historia de la categoría, solo diré que la noción sufrió transformaciones con el paso de los siglos y ganó centralidad nuevamente a partir de la década del 60. Según Castany Prado (2012), primero fue la estabilización del concepto en su versión moderna, el tiempo del "sublime ilustrado" (Burke, Kant), luego habría seguido una etapa romántica (Schelling, Schopenhauer, Nietzsche) y, finalmente, en el siglo XX la categoría habría tenido una nueva vida teórica bajo el signo de la posmodernidad (Lyotard).

Más allá de la vida teórica de la noción, lo sublime parece entrañar un gran poder descriptivo y analítico. Seguramente haya sido esta capacidad analítica lo que explique que distintos autores, por ejemplo, Heinrich Wölfflin (1986) y Arnold Hauser (1978), la hayan empleado para caracterizar estilísticamente el barroco, un período artístico y social previo a la consolidación de la categoría. Lo de Hauser en especial resulta curioso: el autor utiliza el término sublime en su acepción silvestre y no como categoría estética; sin embargo, su descripción del arte barroco (que tiene a Wölfflin como fuente) refiere, entre otras cosas, a la infinitud, la inagotabilidad, el estremecimiento, la "disolución de las formas" o el "eco de los espacios infinitos". Puede verse allí el aparato descriptivo de lo sublime operando en plenitud más allá de la utilización explícita de la categoría

Solo recientemente los game studies han prestado atención a lo sublime (Shinkle, 2010; Martin, 2011; Betts, 2014; Vella, 2015; O’Sullivan, 2017). Se trata mayormente de abordajes teóricos y generalistas que analizan pocos casos. Mientras tanto, la producción del videojuego exhibe cada vez una mayor cantidad y diversidad de formas de exceso. Por ejemplo, ya son varios los juegos que, como Laser League o Furi, suspenden temporalmente la representación tridimensional para colmar la pantalla de formas geométricas, debilitar el referente (es decir, la semejanza) y abstraer la imagen. Hay antecedentes, como los momentos bullet hell de los space shooter, donde la acumulación de enemigos y disparos satura el espacio y produce una coreografía de formas y colores. Se recorta así un sublime visual que se emplaza al nivel de la imagen y que reacciona contra el realismo tridimensional (una actualización del carácter sistémico y tensional de lo sublime y lo bello según Burke).

Pero el videojuego es un medio interactivo, ergódico, que gestiona agenciamientos (Aarseth, 1997, Murray, 1997). Sería extraño que la tendencia hacia el exceso no se asentara a su vez esta dimensión. ¿Existirá algo así como un sublime lúdico, es decir, una forma de exceso que tome a su cargo ya no la visualidad sino la interacción misma?

\section{Dark Souls}

En 2011, From Software lanzó Dark Souls. La saga tendría dos entregas más en 2014 y 2016, además de un antecedente: Demon's Souls (2009). La serie cosechó una reputación en poco tiempo: los juegos eran de una dificultad infrecuente, con un sistema de penalización que castigaba severamente los errores y obligaba a jugar estratégicamente y con gran precisión. 
El lanzamiento de cada nueva entrega fue leído como respuesta a una tendencia de diseño consistente en producir juegos cada vez más sencillos, con niveles de dificultad bajos que no castigaban los errores o, si lo hacían, era de manera amable. Así, fue revelando sus contornos un gran cisma estilístico: de un lado, un perfil de jugador hardcore que busca juegos con desafíos que demanden la adquisición y despliegue de destrezas; del otro, el perfil casual, que prefiere juegos simples y breves que puedan jugarse de manera intermitente y que no requieran casi de habilidades lúdicas previas. Los dos movimientos venían gestándose desde bastante antes, pero la llegada de la serie Souls fue uno de los catalizadores del conflicto. Por ejemplo, la aparición de las interfaces miméticas, como el controlador Wiimote, permitió el ingreso al medio de nuevos jugadores y la estabilización de los party games, que no requerían de experiencias videolúdicas previas (Juul, 2010). Hasta ese momento, en líneas generales, para jugar videojuegos era necesario poseer conjuntos de habilidades específicas que variaban según los géneros (la precisión y la velocidad de los movimientos de los fighting games no se parecían, por ejemplo, a las grandes cantidades de información que debía procesarse y ponerse en obra en los juegos de estrategia por turnos). Terminó generándose un diagnóstico generalizado: los juegos serían cada vez más fáciles y exigirían menos a sus jugadores; esa reconfiguración posibilitaría el acceso de un número mayor de jugador y, para algunos, se volvería una estrategia industrial para maximizar beneficios (Kollar, 2011; Abbott, 2012; Kain, 2012; Krasny, 2013)1. El lanzamiento de juegos como los de la serie Souls fue entendido, entonces, como una respuesta hardcore al nuevo auge de lo casual y al crecimiento de una oferta lúdica complaciente que habría dominado el diseño mainstream. Esto se extiende más allá incluso del terreno casual: la tendencia se habría derramado a géneros como el first-person shooter con novedades como el auto-aim o las grandes cantidades de municiones y recursos disponibles (Meixsell, 2012), y a los juegos de plataformas de Nintendo, que incorporaron modalidades que permitían saltearse automáticamente zonas difíciles (Abbott, 2012)

Las diferencias que trazó Dark Souls con otros juegos de acción son numerosas. Tal vez la más significativa sea la incorporación de una barra de vigor (stamina) que se consume con cada ataque y movimiento. Cuando la barra se agota, el avatar se encuentra inerme ante sus enemigos y debe ganar tiempo para recuperarla. Esto obliga a gestionar de manera precisa un plan de acción, por ejemplo, alternando con cuidado entre ataque y defensa o evasión y, por lo tanto, remodela completamente el contrato del género de rol, donde es común que el avatar pueda atacar y moverse sin restricciones (Clouse, 2011). Otra diferencia significativa fue introducida en el sistema de guardado: hasta la serie Souls, era frecuente que los juegos pudieran ser guardados manualmente por el usuario o, cada vez con mayor frecuencia, que contaran con un sistema de autoguardado que asegurara que no se perdiera el progreso. En Dark Souls hay fogatas que funcionan como checkpoints en los que se puede salvar el estado de la partida. Son escasas, el camino a cada una está poblado de obstáculos y enemigos, y cada vez que se descansa junto a una fogata los enemigos derrotados vuelven a sus posiciones (por lo que la exploración siempre supone una galería estable de peligros). Una mecánica adicional se suma al conjunto: cada vez que el se pierde, se retorna a la última fogata en la que se haya descansado, pero todo lo conse- 
guido hasta el momento permanece en el lugar donde cayó el avatar. El protagonista tiene la chance de volver al sitio y recuperar así su botín, pero si muere nuevamente el botín desaparece para siempre. Este mecanismo suma una dificultad inédita y refuerza la necesidad de planificar con precisión cada movimiento. si se pierden las almas y la humanidad obtenida, se hace todavía más improbable el avance por las áreas siguientes del juego (Castellano, 2011). Según Meixsell (2012), mientras la mayoría de los juegos del momento incrementaba la cantidad de tutoriales y buscaba mecanismos para suavizar las rispideces de la experiencia lúdica y, también, para prevenir la derrota, Dark Souls ofrecía un desafío bruto pero justo, donde la dificultad era abrumadora y sin concesiones.

Estos cambios implicaron un gran desvío respecto del género de rol y la mayor parte de juegos de acción del momento, que solían tener penalidades muy leves, sistemas de combates simples y de uso más o menos libre, y que posibilitaban (incluso sugerían) la exploración libre del entorno. La serie Souls se caracterizó además por el despliegue de una narrativa escasa y difusa, hecha de pistas dispersas y de climas: en busca de explicaciones, la comunidad de jugadores se lanzó a la interpretación del mundo del juego y a la generación de hipótesis narrativas. Otra diferencia sustancial con la oferta del momento, entonces, esta vez respecto de los juegos con relatos tradicionales que incluyen arcos narrativos claros y en los que se provee toda la información narrativa necesaria (Kollar, 2011).

La serie Souls se prolongó con otros juegos del mismo estudio (Bloodborne, 2015; Sekiro: Shadows Die Twice, 2019) y devino una condición de producción de muchos juegos posteriores que incorporaron aspectos como la barra de vigor, limitaciones en el salvado de la partida, penalizaciones severas y complejos sistemas de combate. La irrupción, ascenso y extensión de la serie Souls exhibe los rasgos de una disrupción estilística que afirma una oferta discursiva en tensión con otra. No es raro, entonces, que el estilo Souls haya pasado a formar parte de las preferencias hardcore y que fuera esgrimido como argumento contra la tendencia casual. Los conflictos estilísticos de la producción del medio y de sus modos de apropiación culturales parecen haber catalizado en torno a un aspecto: la complejidad de la experiencia y la demanda de esfuerzo y de destrezas presupuestas (a diferencia de lo que sucede en otros lenguajes, el conflicto estilístico mencionado no incluye, por ejemplo, aspectos relativos a la imagen, el espacio, el sonido o la música).

El exceso que entraña un juego Souls, tanto como la distancia que traza con el resto de la producción, parece responder perfectamente al campo de lo sublime y a su universo de desborde. La singularidad reside, en todo caso, en que el exceso opera aquí en el nivel de la interacción, es decir, que el desborde no es audiovisual sino lúdico. Los rasgos mencionados generan un nivel de resistencia textual muy elevado: todo, tanto la diégesis (enemigos, trampas) como los mecanismos paratextuales (guardado), obstaculiza el avance y abre varios frentes de problemas y habilidades diferentes; el más mínimo error puede infligir pérdidas irreparables. De esta forma, la dificultad resulta abrumadora, excesiva, pero entraña a su vez un placer: sobreponerse a situaciones lúdicas de una gran adversidad por la vía del aprendizaje. Welsh (2011) sostiene que la exposición prolongada a los obstáculos y su eventual superación revaloriza y acreciente cada pequeña recompensa que el juego gestiona. Cada derrota permite retroalimentar ese circuito lúdico: 
You will die, a lot. You will die on the end of a sword, on the edge of an axe, crushed by a boulder, impaled on fangs; you will be poisoned, eaten, stabbed, assassinated and pushed off cliffs. Death is everything in Dark Souls. It's education, it's progress, it's the recurring stylistic and thematic motif that runs through all of its spectacularly varied, decaying and depraved environments (McDonald, 2012)

\section{Wanna be the Guy}

La cuestión del placer asociado al fracaso, sin embargo, no es algo que pueda reducirse a los juegos de estilo Souls. En The Art of Failure (2013), Juul problematiza el asunto: si se parte de la base de que la mayoría de las experiencias lúdicas suponen alguna forma de derrota, y que estas derrotas son (al menos en el videojuego) la norma antes que la excepción, entonces, postula el autor, la fruición de lo lúdico debe estar necesariamente relacionada con el fracaso, y este debe entrañar placeres específicos, más allá de que, en términos sociales, la victoria aparezca como preferible a la derrota. Uno de esos placeres, dice Juul, es el mejoramiento de sí: con cada partida perdida, el jugador es lanzado a un ciclo de repeticiones que le permiten tomar conciencia de sus errores, adquirir nuevas destrezas y perfeccionarlas. El mecanismo que domina de ese circuito es, claro, el aprendizaje: sería ese proceso, y las gratificaciones que conlleva, lo que proporcionaría al videojuego un lugar de centralidad en la cultura contemporánea. La hipótesis de Juul permitiría explicar la popularidad de juegos estilo Souls y sus desafíos desproporcionados: la recompensa por cada pequeño logro estaría a la altura de los obstáculos a superar, por lo que esos juegos proveerían placeres tanto o más intensos que el resto. Pero esa hipótesis falla cuando se la aplica a otros tipos de juegos, por ejemplo, a los casuales: la expansión en el nuevo milenio de juegos de muy baja dificultad, con sistemas de penalización leves o a veces incluso nulos, en muchos casos no contempla la experiencia de fracaso y, por ende, cancela ese tipo de aprendizaje (que, ciertamente, no debe ser el único). Podría concluirse, entonces, que la relación entre fracaso y aprendizaje no puede extenderse a toda la producción del videojuego, aunque sí a una buena parte, en especial a la que se afirma a partir del estilo Souls. Antes de la aparición y de la estabilización del estilo Souls, algunos juegos, realizados por usuarios al margen de la órbita de la industria y los estudios, ya perfilaban una oferta distinta a la mainstream. En I Wanna be the Guy (2007), de Kayin (Michael O'Reilly), un pequeño avatar debe desplazarse a lo largo de una serie de niveles que reenvían a clásicos populares del videojuego de los 80 y 90 (Casella, 2015). A primera vista, la organización responde al género de plataformas: hay que moverse de un punto a otro de la pantalla sorteando obstáculos y enemigos mientras se calibran saltos y acrobacias. Pero a poco de empezado, el juego exhibe un notorio desvío respecto del pacto del género: pudiendo elegir entre dos caminos, uno hacia arriba y otro hacia abajo, se descubre que la ruta inferior supone una dificultad insuperable y que no queda otra opción que tomar el camino superior. Allí, un paisaje bucólico con árboles y manzanas no presenta, en principio, ningún peligro: pero ni bien se pasa debajo de los árboles, una manzana cae y el avatar estalla. La acción recomienza y atravesar el bosque se vuelve una tarea infructuosa: hay 
que descubrir qué manzanas caen, en qué momento lo hacen (si antes o después de pasar debajo de ellas), si caen juntas o de manera alternada. El recorrido puede llevar muchos intentos. Cuando finalmente se llega a la esquina derecha de la pantalla, se puede subir a unas plataformas que conducen al siguiente nivel en el extremo izquierdo. Pero cuando se está saltando de una plataforma a otra (acción elemental del género), una manzana que no había caído antes ahora sube y mata nuevamente al avatar.

Se está ante un fenómeno lúdico diferente del del ascenso de la serie Souls: mientras que allí había una exacerbación de los niveles de dificultad, es decir, del desafío y las penalizaciones, $I W b t G$ muestra un escenario distinto. Ya no se trata de una exacerbación de los rasgos que modelan la dificultad, sino de la diseminación de una serie de trampas y de engaños lúdicos que reorganizan totalmente el contrato semiótico del género de plataformas. Como pudo verse, en la propuesta de los juegos Souls se reconoce, aunque hiperbolizado, el pacto del género de rol, mientras que en $I W b t G$ la naturaleza del contrato enunciativo es diferente: la resistencia textual ya no proviene de un desafío complejo, sino del carácter arbitrario y sorpresivo de los obstáculos (Bastien, 2011).

Acá opera otra modalidad de lo sublime. Burke, cuando lista y comenta los aspectos que pueden llegar a provocar el sentimiento de lo sublime, habla de la dificultad": "Cuando una obra parece haber requerido una fuerza y un trabajo inmensos para llevarse a cabo, la idea es grande" (1998, p. 57). Burke se refiere al esfuerzo invertido en la creación de algo y no a la destreza que pudiera exhibir la factura: por ejemplo, Stonehenge, explica, es sublime debido a la monumentalidad de su organización, y la brusquedad general del conjunto no hace más que reforzar el efecto general.

Breve comentario histórico: con el lanzamiento de Super Mario Bros. en 1985, Nintendo estableció una filosofía de diseño de niveles que habría de afirmarse con cada nueva entrega de la serie. A grandes rasgos, esa filosofía puede resumirse de la siguiente manera: la complejidad debe estar en función de una curva de aprendizaje suave, la introducción de enemigos debe ser gradual y variada, que no debe haber obstáculos que no sean comunicados abiertamente al jugador. De alguna forma, Nintendo acabó fijando un estilo de diseño que dominó prácticamente el género de plataformas. Si hubiera que caracterizar en términos enunciativos esta propuesta, habría que decir que se trata de una enunciación transparente, que trata de borrar sus huellas y dejar en el centro de la escena el despliegue diegético. Se sabe que cada lenguaje presenta diferentes valencias enunciativas (Metz, 1990, Verón, 2005, Pérez Latorre, 2012, Steimberg, 2013). El efecto de transparencia no se construye igual en literatura, el cine o el videojuego, cada lenguaje pone en disponibilidad diferentes insumos discursivos. En el videojuego, una forma posible de funcionamiento de la transparencia (aunque no la única) consiste en privilegiar la fluidez del desarrollo lúdico, es decir, hacer que la trayectoria a través de niveles y objetivos no se vea interrumpida por obstáculos muy pronunciados ni por elementos del entorno que detengan el avance. En este sentido, borrar las huellas de la enunciación supone que el despliegue de la dificultad esté supeditado al fluir lúdico, que no haya elementos que señalen de manera muy evidente el trabajo enunciativo.

$I W b t G$ supone un desvío respecto de esa corriente estilística, que continúa al día de hoy dominando la oferta del género de plataformas. El nivel inicial, con las manzanas que caen o se elevan, condensa la radicalidad de la propuesta: aquí los obstáculos son arbitrarios y 
de una complejidad abrumadora. La dificultad resulta desbordante desde el comienzo, no hay un incremento gradual del desafío, la aparición de los obstáculos es sorpresiva. Algunos recursos se vuelven recurrentes, por ejemplo, los bloques invisibles que interrumpen un salto y envían al avatar hacia un precipicio; o las plataformas que, una vez pisadas, se desploman. Se trata de dos elementos comunes en el género, de hecho, se los puede hallar en la mayor parte de los juegos de Mario, pero la manera de gestionarlos es totalmente distinta: en los juegos de plataformas tradicionales, la dosificación de las trampas, así como su comunicación, se da siguiendo los mandatos del estilo de Nintendo, donde el fluir lúdico es gradual y el incremento de la dificultad adopta la forma de una curva muy suave. El sinfín de trampas de $I W b t G$ no hace otra cosa que llamar la atención sobre la enunciación, es decir, sobre la instancia dadora del texto. Nuevamente, el ataque de las manzanas resulta revelador: un elemento del entorno, aparentemente decorativo, sin incidencia lúdica, acaba siendo un peligro muy difícil de sortear. Esto interrumpe cualquier posibilidad de flujo lúdico estable; la dificultad y la aparición inesperada del obstáculo muestran el trabajo de la organización textual, el juego se revela en tanto un quehacer discursivo. El quiebre de $I W b t G$ respecto de las previsibilidades del estilo dominante en el género de plataformas, entonces, podría resumirse de la siguiente manera: hay un enunciador activo que dispone claramente los mecanismos textuales, que hace mucho (Verón, 2005) y que, por lo tanto, revela la labor del texto. En $I W b t G$, esta opacidad enunciativa se acentúa además por el caudal de reenvíos a juegos y géneros del pasado, sobre todo de los 80 , en muchos casos con referencias directas que incluyen personajes, espacios y convenciones lúdicas. Esta autorreferencialidad y la multiplicidad de las referencias y guiños visibiliza todavía más la enunciación y su trabajo.

La propuesta enunciativa de IWbtG se expandió a numerosos juegos, por ejemplo, a $\mathrm{N}+$ (2008), Super Meat Boy (2010) y VVVVVV (2010) (Bastien, 2011) y, más recientemente, a Celeste (2018): todos juegos que se apartaron del estilo tradicional en el género de plataformas para ofrecer en su lugar desafíos desmesurados. La insistencia en el tiempo de estos rasgos señala la vigencia de un estilo que, si bien marginal, ha modificado el paisaje del género. Si hubiera que caracterizar la desmesura de estos juegos, habría que hablar de un exceso enunciativo: la dificultad sobreabundante que surge de la diseminación de trampas y obstáculos muy difíciles de franquear indican la presencia del enunciador, y de uno especialmente activo, laborioso, cuyo trabajo moviliza a gran escala los insumos textuales. En general, las pocas veces que los games studies problematizaron la enunciación del medio, lo hicieron atendiendo exclusivamente al enunciatario, es decir, al jugador-modelo, o por lo menos lo privilegiaron por sobre el enunciador (de Mul, 2005; Perez Latorre, 2012, Vella, 2016, Maté, 2019a). La expansión de juegos como IWbtG plantea dos cuestiones: uno, la práctica inexistencia de estudios enunciativos sobre el videojuego y, en consecuencia, la ausencia de instrumentos y métodos para dar cuenta de un fenómeno enunciativo singular cuyo funcionamiento parece descansar en la exacerbación de la instancia dadora del texto. Dos, que tal vez uno de los principales atractivos de juegos como $I W b t G$ haya que buscarlo en esa propuesta discursiva particular, en el contacto con un juego que quiebra con la tradición de la transparencia y elabora visiblemente una figura de enunciador activo, exuberante, omnipotente. 


\section{Kaizo Mario}

Casi al mismo tiempo que $I W b t G$, circuló ampliamente un rom hack del juego Super Mario World. El mod fue atribuido a un tal T. Takemoto, cuya identidad nunca se reveló, y recibió el título de Kaizo Mario (2007), más sencillo que el original en japonés. Para ese momento existía una robusta comunidad de modding que se dedicaba a modificar por diferentes vías juegos de la serie Mario para luego compartirlos online y jugarlos. Kaizo Mario tuvo un gran éxito dentro de la comunidad, trascendió rápidamente sus límites y se transformó en poco tiempo en uno de los hacks más populares de la escena, al punto de haber originado una designación estilística: de allí en más, los hacks de Mario que tuvieran grados semejantes de complejidad serían identificados con el término Kaizo.

El mayor atractivo del juego fue su elevadísimo nivel de dificultad. A diferencia del estilo tradicional que informó (e informa) a la mayor parte de la producción del género de plataformas, Kaizo Mario entraña un desafío de una complejidad sin precedentes. El juego exige un timing perfecto, un despliegue milimétrico de saltos y movimientos a lo largo de niveles poblados de trampas y enemigos. Sin embargo, se está ante un fenómeno distinto al de $I W b t G$. Los dos son juegos que requieren de una gran cantidad de habilidades que rebasa claramente el contrato discursivo habitual del género de plataformas, pero mientras que los niveles sucesivos de $I W b t G$ son en general variaciones de un mismo conjunto de obstáculos y trampas desplegados de manera sorpresiva, Kaizo Mario exhibe un abanico de operaciones lúdicas diferente.

Esto es algo que caracterizó a la escena de hacking de juegos de Mario: la apropiación mediante herramientas tecnológicas diversas y la transformación de los juegos originales en insumos disponibles para ser reconfigurados, parece haber provisto un espectro de variaciones lúdicas prácticamente inagotable. Esto puede verse también en muchos de los niveles creados por jugadores en Super Mario Maker (2015) y Super Mario Maker 2 (2019), editores oficiales lanzados por Nintendo: el repertorio de estrategias de diseño abierto por los niveles creados por usuarios, muchos de ellos de una complicadísima ingeniería, muestra un rango expresivo enorme que recupera tanto como reinventa y subvierte el estilo de Nintendo. Un tipo de diseño ya estabilizado consiste, justamente, en la elaboración de niveles de una dificultad muy elevada. Estos niveles suelen ganar popularidad en las redes, reconocimiento de la comunidad de jugadores y ser comentados en la prensa especializada; se los identifica, además, bajo la rúbrica estilística Kaizo.

Tanto en los Kaizo Marios como en los niveles generados con Super Mario Maker, por momentos la dificultad llega a niveles tan elevados que se produce un desplazamiento de género, que va del de plataformas al de puzzle (llamado a veces de ingenio en español). Véase, por ejemplo, el nivel 9 de Kaizo Mario World 3. La primera fase transcurre dentro de lo esperado: el nivel exige una coreografía imposible a través de tubos, plataformas que se caen, enemigos y la utilización quirúrgica de unos pocos ítems que ofrece el escenario. Pero en la segunda fase, algo cambia. El nivel queda dividido en dos espacios bien diferenciados: arriba y abajo. Cada uno posee su dinámica propia: abajo, el avatar debe desplazarse y efectuar el repertorio habitual de acrobacias; arriba, una caparazón de tortuga se mueve a través de un artefacto enrevesado. La superación de la fase requiere que el avatar 
active y dirija, mediante mecanismos que hacen de controles ubicados en la zona inferior, la trayectoria de la caparazón en el área superior: solo la trayectoria correcta permite la superación de la fase. Ahora bien, el desafío no se reduce solo a superar los obstáculos en la zona inferior, sino al desciframiento de, por un lado, los mecanismos que permiten controlar el recorrido de la capazón y, por otro, el timing necesario en el que debe ejecutarse cada acción. De esta forma, el pacto discursivo muta: las habilidades con las que la enunciación inviste a su enunciatario ahora contemplan además aptitudes cognoscitivas, de puesta en obra de facultades de interpretación y desciframiento. En otras palabras, la complejidad es tal que la dificultad incorpora ahora el repertorio de desafíos de otro género, el puzzle. Puede verse así que algunos momentos de Kaizo Mario muestran una exacerbación mayor incluso a la de $I W b t G$ : mientras que el juego de Kayin supone una radicalización de la oferta semiótica del género de plataformas, el de T. Takemoto reorganiza totalmente su escena discursiva al superponer dos géneros y desplegar un desafío bifronte. El juego de plataformas ha entrado en contacto con otros géneros en más de una ocasión. De hecho, por la misma época en que se conocía Kaizo Mario World, aparecía Braid (2008), de Jonathan Blow, cuya novedad se cifraba en una cruza inédita entre plataformas y puzzle. Pero se trata de una mixtura muy diferente a la de Kaizo Mario: mientras que en sus momentos más complejos,este obliga a poner en obra al mismo tiempo destrezas características de los dos géneros, Braid supedita las mecánicas de plataformas a las de puzzle, logrando así un desafío balanceado y específico. De los dos géneros presentes, uno domina la escena.

Con el lanzamiento de Super Mario Maker 1 y 2 se dio un fenómeno singular: muchos de los niveles creados y compartidos por los usuarios no solo se apartaban del rango estilístico de Nintendo, sino que con frecuencia abandonaban completamente el género de plataformas y adoptaban funcionamientos semióticos extralúdicos, como sucede, por ejemplo, con los niveles calculadora (Sheridan, 2019), en los que no se juega. De ese inmenso caudal de niveles creados por usuarios surgió una nueva tendencia: niveles pequeños, breves, que modificaban el funcionamiento del género de plataformas hasta transformarlo en un puzzle. Por ejemplo, en el nivel One Way Clip, del usuario Deakula, Luigi se encuentra encerrado en un cuarto. El camino a la puerta de salida está bloqueado y no hay una manera directa de alcanzarla. Hay además un escarabajo y una especie de aparato en la esquina superior izquierda. La solución es la siguiente: Luigi debe tomar el escarabajo, dirigirse hacia el aparato y depositarlo allí; un glitch, es decir, un error de programación, hace que el escarabajo atraviese el tubo de la máquina y active un bloque que posibilita el descenso de un hongo. Ahora Luigi debe posicionarse justo encima de la puerta y esperar a que el hongo llegue junto a él: de esa forma, cuando el avatar incrementa su tamaño, se activa otro glitch que le permite deslizarse por la cinta que lo separa de la puerta, alcanzarla y superar el nivel (Hernandez, 2019). Las competencias exigidas no tienen que ver con el despliegue de acrobacias en el espacio (como en el género de plataformas), sino con el desciframiento de un enigma. Así, los personajes, el espacio y las acciones de un género se vuelven insumos de otro, el género de puzzle. Pero se trata, además, de un puzzle donde la solución no puede surgir simplemente de la prueba y el error ni del contacto con el entorno: el acertijo demanda un conocimiento específico de los glitches $^{3}$ del juego y su correcta implementación. Danesi (2002) explica que todo puzzle 
convoca saberes previamente adquiridos: estos nuevos niveles realizados con el editor Super Mario Maker reclaman interpretantes de una gran especificidad, conocimientos que hacen a la serie de juegos Mario y a sus aspectos menos conocidos.

La exacerbación de los niveles de dificultad parece haber conducido a nuevas formas de producción amateur: primero, los rom hack cada vez más complejos terminan estabilizando una oferta estilística nueva, los juegos Kaizo, en los que los usuarios, por medio de herramientas tecnológicas diversas, se apropian de los juegos de Mario y los remodelan hasta desfigurar completamente el contrato discursivo del juego de plataformas. Segundo, con el lanzamiento de los editores oficiales de Nintendo, se publican niveles que se apartan por completo del funcionamiento del género de plataformas y, en cambio, pasan a operan de acuerdo con las reglas del puzzle. Los excesos lúdicos, en este caso, terminaron de lanzar la escena de rom hacking y de creación de contenidos por usuarios hacia nuevas tendencias que reinventan la oferta semiótica del género de plataformas.

Sicart y Wilson incluyen los Kaizo Mario dentro de un estilo de diseño que denominan abusive game design, que cifraría su propuesta en el despliegue de escenas lúdicas que favorecen diferentes formas de malestar. Este nuevo estilo, explican, surgiría como reacción contra la corriente de defensa del jugador (player advocacy), dominante dentro del diseño mainstream, que propone vías para mantener cautiva la atención y el interés del jugador minimizando la frustración. Los autores sostienen que, mientras la corriente de defensa del jugador pregona un tipo de diseño centrado en el gameplay, el diseño abusivo, en cambio, supone un diálogo entre diseñador y jugador, debido a que el diseño se evidencia como tal y, en consecuencia, se explicita una forma de intercambio; la mano del diseñador acabaría revelándose en los mecanismos de juego y, por lo tanto, interpelaría al jugador. Esa idea de diálogo que señalan Sicart y Wilson, si bien refiere a un intercambio empírico, entre sujetos, puede utilizarse para caracterizar enunciativamente la escena discursiva de los Kaizo Mario. La visibilización de un "diálogo", una vez roto el diseño tradicional centrando en el gameplay, parece estar indicando un desplazamiento enunciativo: de una escena transparente, donde la enunciación se borra, se pasa a otra mostrada, en la que las huellas del enunciador se vuelven ostensibles y no hacen más que señalar el trabajo del texto. Desde este punto de vista, la novedad de lo que se llamó diseño abusivo no es otra cosa que la emergencia de una nueva forma de opacidad enunciativa en el espacio de un medio que parece haber dominado la transparencia.

A su vez, algunos trabajos sobre puzzles dentro y fuera del videojuego llaman la atención acerca de su dimensión comunicativa en términos parecidos a los de Sicart y Wilson. Arnott (2012) explica que el puzzle supone siempre un intercambio imaginario entre el jugador y el desarrollador: suele pensarse que son juegos mayormente solitarios, dice el autor, pero, a diferencia de lo que sucede con otras formas lúdicas, aquí cada movimiento, cada decisión del jugador lo confronta con un mundo claramente diseñado, que exhibe la mano de un creador. En la misma línea, Tronstad (2005) recoge diferentes investigaciones que coinciden en que el puzzle, en sus distintas formas (lúdica, videolúdica o literaria, por ejemplo), supone siempre una "situación social", una interacción simbólica entre un riddler y un riddlee. Para Tronstad, el puzzle exhibe siempre la presencia necesaria de su creador, "the one who knows the answer". Al igual que se vio con los planteos de Sicart y 
Wilson, también estas observaciones pueden leerse en términos enunciativos: a diferencia de otras formas lúdicas, el puzzle señalaría con énfasis el trabajo de un enunciador cuyos rastros permanecen en el despliegue del enigma a elucidar. Este fragmento de Perec resulta esclarecedor:

Despite appearances, puzzling is not a solitary game: every move the puzzler makes, the puzzle-maker has made before; every piece the puzzler picks up, and picks up again, and studies and strokes, every combination he tries, and tries a second time, every blunder and every insight, each hope and each discouragement have all been designed, calculated, and decided by the other. (citado en Arnott, 2012, p. 435)

De esta manera, el desplazamiento genérico de los Kaizo Mario, del juego de plataformas al de puzzle, entraña un movimiento ahora comprensible: la búsqueda de desafíos de una dificultad mayor, con el consiguiente despliegue de una figura de enunciador cada vez más visible, condujo el flujo de apropiaciones hacia el terreno del puzzle, forma lúdica que parece haber funcionado siempre bajo un régimen de opacidad, es decir, donde el enunciador, lejos de borrar los rastros de su trabajo (como sucede en el diseño mainstream) exhibe necesariamente la huella de su actividad, y donde esta visibilización discursiva debe haber posibilitado (seguramente) placeres lúdicos singulares (la vida social del puzzle, larga y múltiple, así lo sugiere).

Una vez más, conviene recordar que una de las dimensiones de lo sublime para Burke es en la dificultad, entendida como el esfuerzo que denota la factura de un objeto. La alambicada ingeniería lúdica de los Kaizo Mario llama la atención sobre la complejidad de su propio diseño: el grado de elaboración y de detalles de esos juegos convoca la excesividad y el sobrecogimiento. No es casual que uno de los pilares de la comunidad de rom hacking sea la búsqueda de nuevas soluciones de diseño que permitan la creación de desafíos cada vez más abigarrados. De los casos vistos antes (el estilo Souls y $I W b t G$ ), puede decirse que estos juegos participan del mismo tipo de sublimidad, aunque con gradaciones.

Cuando se habla de los Kaizo Mario se pasa a analizar el reconocimiento: se trata de apropiaciones por parte de usuarios que remodelan completamente una oferta textual preexistente. La regularidad de estas prácticas permite hablar de una gramática de reconocimiento, un conjunto de reglas que organiza con cierta previsibilidad la formas de la recepción. Esto permite sugerir, a su vez, otra dimensión de exceso: la que surge de los videos en los que jugadores muestran partidas cercanas a las perfección. La expansión de los Mario Kaizo se dio en paralelo con la estabilización un fenómeno paratextual: los videos en los que usuarios juegan niveles de gran dificultad. Esos videos son parte integral del fenómeno Kaizo, y si bien su variedad resulta imposible de sintetizar, puede pensarse, a grandes rasgos, en dos tipos: uno, videos en los que los usuarios juegan y pierden muchas veces, y que por lo general buscan un efecto cómico (el jugador se somete voluntariamente a la burla pública); dos, videos en los que usuarios juegan niveles Kaizo evidenciando un gran virtuosismo que les permite sortear casi sin fallas desafíos de una dificultad abrumadora. Nos interesa el segundo tipo, ya que ahí se activa una vía de lo sublime, pero ahora la 
dificultad (en términos burkeanos) no se ubica en la complejidad del diseño, sino en la sofisticación de la performance. El exceso, esta vez, no hay que buscarlo en la factura del nivel, sino en su superación, en el despliegue de destrezas, en el trazado en la imagen de una secuencia exacta de movimientos que conduce a superar la totalidad de los obstáculos; una coreografía de una precisión impresionante que produce asombro y deslumbramiento.

\section{Conclusiones}

Los casos trabajados, y la tendencia que dibuja cada uno, señalan diferentes modalidades del exceso en el videojuego. Estas modalidades pueden comprenderse mejor a la luz de la categoría de lo sublime, que desde diferentes campos del saber, y aplicada a distintas áreas de la cultura, ha sido utilizada con recurrencia para analizar formas de desborde. Como pudo verse, los casos se inscribían con nitidez en los dos polos de la circulación: producción (estilo Souls, $I W b t G$ ) y reconocimiento (Kaizo Marios, videos con perfomances lúdicas de esos mismos juegos). En las dos instancias se verifican desbordes, excesos que no son otra cosa que el rebasamiento de ciertas normas, de ciertas gramáticas preexistentes: de las reglas que organizaron (y organizan) una buena parte de la oferta semiótica del videojuego (estilo Souls, IWbtG, Kaizo Marios); de lo que se espera de una performance lúdica promedio, con un espectro elevado de errores y fracasos (videos de partidas perfectas de niveles Kaizo). En todos los casos, lo sublime surge de la complejidad del conjunto, del trabajo invertido en las partes y en su su interrelación, sin importar que se hable del diseño de un nivel o de una performance lúdica. Uno y otro quiebran sus previsibilidades respectivas y construyen discursivamente asombro, recogimiento. El quiebre respecto de un conjunto de previsibilidades es decisivio: es esa tensión lo que posibilita el despliegue del exceso, el desborde de una norma previa. Debe recordarse que, en muchas de sus conceptualizaciones (por ejemplo, en la de Burke y la de Kant), lo sublime operó siempre en relación a otra cosa, con frecuencia, lo bello. Lo bello en Burke y Kant, dos autores que ayudaron a fijar una discursividad de lo sublime, supone nociones muy distintas, pero esa diferencia no es significativa aquí, lo que importa es subrayar el carácter sistemático de lo sublime, su relación diferencial con otros conceptos.

En el presente, el videojuego mantiene toda clase de intercambios con otras esferas culturales, en especial con el arte. Los primeros cruces tuvieron formas concretas: ingreso a los museos, apropiación por parte de artistas, categoría de art game. El paso de los años parece haber expandido esas vías: un fenómeno todavía menos visible, menos estruendoso, pero también de gran vitalidad, es la actualización en la producción del videojuego de categorías estéticas como las de lo sublime o lo siniestro. Esto marca, tal vez, la aparición y posible estabilización de una nueva vía de intercambios con el campo de las artes, y que consistiría en la apropiación por parte del videojuego de conjuntos de operaciones asociadas históricamente a la producción de los lenguajes artísticos. 


\section{Notas}

1. En el presente, esta tendencia genera grandes cambios: muchos estudios disminuyen considerablemente la dificultad de sus juegos si la comunidad de seguidores se pronuncia sobre el tema en redes y webs. Ya no se trata solamente de una merma generalizada de la dificultad, sino que un juego puede llegar a sufrir grandes modificaciones lúdicas después de su lanzamiento (Alexandra, 2017).

2. Sobra la aclaración: cuando habla de dificultad, Burke no piensa la dificultad en términos lúdicos, sino en el esfuerzo que denota un objeto.

3. Es un tipo de glitch muy común en muchos juegos. Se lo suele llamar wall clip, y se caracteriza por permitir el tránsito a través de paredes y de espacios cerrados que normalmente no habilitan la circulación.

\section{Bibliografía}

Aarseth, E. (1997) Cybertext: Perspectives on ergodic literature. Baltimore: John Hopkins University Press.

Alexandra, H. (17 de junio de 2017) More Video Games Are Getting Easier After Release. Kotaku. Recuperado de: https://kotaku.com/more-video-games-are-getting-easier-afterrelease-1796459663

Abbott, M. (21 de febrero de 2012) Video Games Are Easier Than Ever, Yet Harder To Manage. Kotaku. Recuperado de: https://kotaku.com/video-games-are-easier-than-everyet-harder-to-manage-5887020

Arnott, L. (2012) Unraveling Braid: Puzzle Games and Storytelling in the Imperative Mood. Bulletin of Science, Technology \& Society, 32(6), 433-440.

Bastien, R. (30 de noviembre de 2011) Reseña Indie: I Wanna be the Guy. Desafío inalcanzable. Atomix. Recuperado de: https://atomix.vg/resena-indie-i-wanna-be-the-guy\%E2\%80\%93-desafio-inalcanzable/

Burke, E. (1998) De lo sublime y de lo bello. Barcelona: Altaya.

Casella, P. (15 de septiembre de 2015) I wanna be the guy, un desafío que no es para cualquiera. Geeky. Recuperado de: https://geeky.com.ar/i-wanna-be-the-guy-un-desafioque-no-es-para-cualquiera/

Castany Prado, B. (2012) Sublimidad y nihilismo en la cultura del Barroco. Revista de Filosofía (37) 2. 91-110.

Castellano, A. (3 de octubre de 2011) Análisis de Dark Souls. Juegos 3D. Recuperado de: https://www.3djuegos.com/juegos/analisis/9592/0/dark-souls/

Clouse, J. (14 de octubre de 2011) Dark Souls Review. The Escapist. Recuperado de: https:// v1.escapistmagazine.com/articles/view/video-games/editorials/reviews/9171-DarkSouls-Review

Danesi, M. (2002) The Puzzle Instinct. The Meaning of Puzzles in Human Life. Indiana: Indiana Press University. 
Donovan, T. (2010) Replay: The history of video games. East Sussex: Yellow Ant.

Gras, M. (1998) Introducción. De lo sublime y de lo bello. Barcelona: Altaya.

Hernandez, P. (15 de julio de 2019) These impossible Mario Maker 2 levels can only be beaten with glitches. Polygon. Recuperado de: https://www.polygon.com/2019/7/15/20694758/ mario-maker-2-tricks-glitches-impossible-levels

Hauser, A. (1978) Historia social de la literatura y el arte. Barcelona: Labor.

Herman, L. (1993) Phoenix: The Fall \& Rise of Video Games. Springfield: Rolenta Press.

Herz, J. (1997) Joystick Nation: How videogames ate our quarters, won our hearts, and rewired our minds. Boston: Little Brown.

Juul, J. (2010) A casual revolution. Reinventing Video Games and Their Players. Cambridge: MIT Press.

Kain, E. (28 de junio de 2012) Games Are Getting Easier: 5 Reasons That's A Bad Thing. Forbes. Recuperado de: https://www.forbes.com/sites/erikkain/2012/06/28/games-aregetting-easier-5-reasons-thats-a-bad-thing/\#7fb619386e8e

Kent, S. (2001) The Ultimate History of Video Games: From Pong to Pokemon. The Story behind the Craze That Touched Our Lives and Changed the World. Rocklin: Prima Communications.

Kollar, P. (3 de octubre de 2011) Dark Souls Review: Dead And Loving It. Game Informer. Recuperado de: https://www.gameinformer.com/games/dark_souls/b/ps3/archive/2011/10/03/dark-souls-review-dead-and-loving-it.aspx

Krasny, A. (6 de octubre de 2013) Are video games getting easier? Agree or Die. Recuperado de: https://agreeordie.com/are-video-games-getting-easier/

Maté, D. (2019a) Estrategias enunciativas del videojuego. Cuatro figuras. InMediaciones de la Comunicación (14) 1.129-150. Recuperado de: https://revistas.ort.edu.uy/inmediacionesde-la-comunicacion/article/view/2889/2929

Maté, D. (2019b) Modalidades de lectura de lo siniestro desde el glitch en el videojuego: viejos temores, nuevos placeres. Revista Chilena de Semiótica, 11. 166-182.

Maté, D. (2019c) Al final del pasillo. Modalidades de lo siniestro en el videojuego. Boletín de Arte (BOA), 19. 1-17.

Martin, P. (2011) The Pastoral and the Sublime in Elder Scrolls IV: Oblivion. Game Studies (11) 3. Recuperado de: http://gamestudies.org/1103/articles/martin

McDonald, K. (30 de septiembre de 2011) Dark Souls Review. IGN. Recuperado de: https:// www.ign.com/articles/2011/09/30/dark-souls-review

Meixsell, J. (31 de diciembre de 2012) Are video games easier than they used to be? Venture Beat. Recuperado de: https://venturebeat.com/community/2012/12/31/are-video-gameseasier-than-they-used-to-be/

Metz, C. (1990) Lénonciation impersonnelle, ou le site du film. París: Méridien-Klincksieck. de Mul, J. (2005) The game of life: Narrative and ludic identity formation in computer games. Raessens, J; Goldstein, J. H. (editores) Handbook of computer game studies. Cambridge: MIT Press. 251-266.

Murray, J. (1997] Hamlet en la holocubierta: El futuro de la narrativa en el ciberespacio. Barcelona: Paidos. 
Pérez Latorre, O. (2012) El lenguaje videolúdico: Análisis de la significación del videojuego. Barcelona: Laertes.

Sheridan, C. (7 de agosto de 2019) Somebody made a working calculator in Super Mario Maker 2 and it's so beautiful. Games Radar. Recuperado de: https://www.gamesradar. com/super-mario-maker-2-calculator

Shinkle, E. (2010) Video Games and the Technological Sublime. Tate Papers, 14. Recuperado de: https://www.tate.org.uk/research/publications/tate-papers/14/video-games-and-thetechnological-sublime

Steimberg, Oscar (2013) Naturaleza y cultura en el ocaso (triunfal) del periodismo amarillo. Semióticas: Las semióticas de los géneros, de los estilos, de la transposición. Buenos Aires: Eterna Cadencia.

Tronstad, R. (2005) Figuring the Riddles of Adventure Games. Paper presentado en Aesthetics of Play Conference. Bergen: Noruega. Recuperado de: https://www.aestheticsofplay. org/papers/tronstad2.htm

Vella, Daniel (2015) No Mastery Without Mystery: Dark Souls and the Ludic Sublime. Game Studies (15) 1. Recuperado de: http://gamestudies.org/1501/articles/vella

-----(agosto de 2016) 'Who Am 'I' in the Game?': A Typology of the Modes of Ludic Subjectivity. Ponencia presentada en DiGRA/FDG '16 - First International Joint Conference of DiGRA and FDG, Digital Games Research Association and Society for the Advancement of the Science of Digital Games, Dundee, Escocia. Recuperado de: http:// www.digra.org/digital-library/publications/who-am-i-in-the-game-a-typology-of-themodes-of-ludic-subjectivity/

Verón, E. (2005) Cuando leer es hacer: la enunciación en la prensa gráfica. Fragmentos de un tejido. Barcelona: Gedisa.

Welsh, O. (3 de octubre de 2011) Dark Souls. Tough Love. Eurogamer. Recuperado de: https://www.eurogamer.net/articles/2011-10-03-dark-souls-review

Wölfflin, H. (1986) Renacimiento y barroco. Barcelona: Paidós.

Abstract: In recent years, some recurrent forms of excess have settled in the video game.
An already common modality involves the exacerbation of the ludic dimension: games
propose challenges that are increasingly difficult to overcome. The tension between this
new production and the mainstream offer suggests the stabilization of operations related
to overflow. In other realms of culture, operations of this type have been analysed in the
light of the aesthetic category of the sublime, mainly since the baroque in artistic langua-
ges. An area of independent video game production is conveying ludic excesses through
different textual strategies, as seen with the expansion of Souls style, I Wanna be the Guy
or Kaizo Mario games. From a semiotic perspective, the main innovation of these games
is related to the regularization of an opaque enunciation that breaks with the expectations
of the genres and the mainstream design and makes the discursive mechanisms explicit. 
This opacity is particularly evident in the visibility of an omnipresent enunciator, whose work is constantly revealed in the overabundance of challenges, traps and puzzles that the games deploy. This communicative displacement is a great novelty in the medium's offer, where traditionally enunciative transparency seems to have dominated.

Keywords: enunciation - Dark Souls - excess - sociosemiotics - perfomance - Kaizo Mario - abusive game design.

Resumo: Nos últimos anos, algumas formas recorrentes de excesso se instalaram no videogame. Uma modalidade já comum envolve a exacerbação da dimensão lúdica: jogo após jogo, são propostos desafios que são cada vez mais difíceis de superar. A tensão entre esta nova produção e a oferta principal sugere a estabilização das operações relacionadas com o transbordo. Em outras esferas da cultura, operações deste tipo foram analisadas à luz da categoria estética do sublime, especialmente a partir do barroco nas linguagens artísticas. Uma área de produção independente de videojogos está a transmitir excessos lúdicos através de diferentes estratégias textuais, como se viu com a expansão de Souls, I Wanna be the Guy ou Kaizo Mario style games. De uma perspectiva semiótica, a principal novidade destes jogos tem a ver com a regularização de um enunciado opaco que rompe com a previsibilidade dos géneros e o design mainstream e torna os mecanismos discursivos ostensivos. Esta opacidade encontra-se principalmente na visibilidade de um enunciador omnipresente, cujo trabalho se revela constantemente na superabundância de desafios, armadilhas e enigmas que os jogos exibem. Este movimento comunicativo é uma grande novidade na oferta do meio, onde a transparência tradicionalmente enunciativa parece ter dominado.

Palavras chave: enunciação - Dark Souls - excesso - sociossemiótica - performance, Kaizo Mario - abusive game design

[Las traducciones de los abstracts fueron supervisadas por el autor de cada artículo] 
